\title{
Particle velocity measurement of binary mixtures in the riser of a circulating fluidized bed by the combined use of electrostatic sensing and high-speed imaging
}

\author{
Wen-Biao Zhang ${ }^{1} \cdot$ Tian-Yu Wang ${ }^{1} \cdot$ Yao-Yao Liu ${ }^{1} \cdot$ Wen-Bo Zhan ${ }^{2} \cdot$ Chi-Hwa Wang ${ }^{3}$
}

Received: 17 December 2019 / Published online: 20 April 2020

(c) The Author(s) 2020

\begin{abstract}
The flow dynamics of binary particle mixtures in the fluidized bed needs to be monitored in order to optimize the related industrial processes. In this paper, electrostatic sensing and high-speed imaging are applied to measure the velocities of polyethylene and sand particles in the binary particle mixtures in fluidization. Experimental studies were conducted on a lab-scale cold circulating fluidized bed. Correlation function between electrostatic signals from upstream and downstream electrodes placed along the riser shows two peaks that represent transit times for the two types of particles. To verify the above results, high-speed imaging was adopted to capture the flow images of particle mixtures. Particle Image Velocimetry and Particle Tracking Velocimetry algorithms were utilized to process the resulted images in order to measure the velocities of polyethylene and sand particles. The reasons for two-peak correlation functions are illustrated based on the frequency spectrums of the mono-solid-phase electrostatic signals and the velocity difference between polyethylene and sand particles. Finally, comparisons on the velocities obtained from electrostatic sensing and high-speed imaging demonstrate the electrostatic sensor can roughly estimate the particle velocity of binary particle mixtures in the near wall region of the circulating fluidized bed.
\end{abstract}

Keywords Binary mixtures $\cdot$ Fluidized bed $\cdot$ Electrostatic sensor $\cdot$ High-speed imaging

$\begin{array}{ll}\text { Abbreviations } \\ \text { ADC } & \text { Analog to digital converter } \\ \text { BFB } & \text { Bubbling fluidized bed } \\ \text { CFB } & \text { Circulating fluidized bed } \\ \text { DCC } & \text { Direct cross-correlation } \\ \text { ECT } & \text { Electrical capacitance tomography } \\ \text { FFT } & \text { Fast fourier transform } \\ \text { PE } & \text { Polyethylene } \\ \text { PIV } & \text { Particle image velocimetry }\end{array}$

Edited by Xiu-Qiu Peng

Chi-Hwa Wang

chewch@nus.edu.sg

1 School of Control and Computer Engineering, North China Electric Power University, Beijing 102206, China

2 NUS Environmental Research Institute, National University of Singapore, Singapore 138602, Singapore
PTV Particle tracking velocimetry

RPT Radioactive particle tracking

\section{List of symbols}

$C(d) \quad$ Cross-correlation coefficient

$f(x, y) \quad$ Gray distribution of the binary image

$L \quad$ Distance between the upstream and the downstream electrodes, $m$

$m_{\mathrm{pq}} \quad$ 2-D moment of order $(\mathrm{p}+\mathrm{q})$

$n \quad$ Strictness of the filter

$S_{1}\left(z_{1}, t\right) \quad$ Upstream electrostatic signals, V

$S_{2}\left(z_{2}, t\right) \quad$ Downstream electrostatic signals, V

$S_{\mathrm{PE}}(t) \quad$ Components from PE particles, $\mathrm{V}$

$S_{\text {sand }}(t)$ Components from sand particles, V

$T \quad$ Integral time, $\mathrm{s}$

$t_{\text {lower }} \quad$ Lower velocity threshold, $\mathrm{m} / \mathrm{s}$

$t_{\text {upper }} \quad$ Upper velocity threshold, $\mathrm{m} / \mathrm{s}$

$\bar{u} \quad$ Mean velocity, $\mathrm{m} / \mathrm{s}$

$\bar{x} \quad X$ coordinates of the centroid

$\bar{y} \quad Y$ coordinates of the centroid

3 Department of Chemical and Biomolecular Engineering, National University of Singapore, Singapore 117585, Singapore 


\section{Greek letters}

$\tau \quad$ Time delay, $\mathrm{s}$

$\sigma_{u} \quad$ Standard deviation of the velocities, $\mathrm{m} / \mathrm{s}$

\section{Introduction}

Due to the advantages of homogenous mixing, high heat and mass transfer rate and easy control, fluidized bed is applied in various industrial fields, such as petroleum, chemical and energy processes (Van Der Hoef et al. 2006). For example, circulating fluidized bed boiler is widely adopted for the co-firing of petroleum coke and coal (Chen and $\mathrm{Lu} 2007$; Duan et al. 2017; Cui et al. 2018). In actual industrial processes, inert particles such as sand are often added to the bed to assist the fluidization and promote the heat and mass transfer. As a result, the flow characteristics of binary particle mixtures in the bed should be investigated to deepen the understanding and further optimize the related industrial processes.

Particle segregation caused by different particle densities and sizes is the major problem of binary particle mixtures in the fluidized bed. The hydrodynamic behavior and segregation of binary particle mixtures with disparate sizes, as well as the influence of particle composition and operation condition in a gas-solid fluidized bed were examined by both laboratory experiments and computational modeling (Gao et al. 2008). Chew et al. (2011a, b) investigated the segregation of binary mixtures and cluster characteristics of Group B particles with continuous size distributions in the dilute riser flow. The mixing behaviors of binary mixtures comprising spherocylindrical particles and spherical particles in a fluidized bed were evaluated using the computational fluid dynamic-discrete element method (Ren et al. 2019). The above researches were mainly focused on the segregation and mixing behaviors of particles in the fluidized bed, whereas, the particle velocity of binary particle mixtures in the fluidized bed has not been fully investigated.

A number of methods have been developed in order to investigate the flow dynamics of solid particles in the fluidized bed, including electrostatic method, acoustic measurement, direct visualization, tomography method, particle tracking and pressure fluctuation measurements (van Ommen and Mudde 2008; Sun and Yan 2016). Due to the complexity associated with the binary particle mixtures, few methods have been applied to measure the flow behavior of binary particle mixtures in a fluidized bed. Upadhyay and Roy (2010) investigated the hydrodynamics of binary mixtures in the fluidized bed using Radioactive Particle Tracking (RPT) and dual source gamma ray densitometry. The effects of mixture composition and air inlet velocity on radial and axial distributions of solids were studied. In order to validate the numerical models, fiber optic and RPT techniques were applied to analyze the flow dynamics of large spherical and cylindrical biomass particles in a bubbling fluidized bed (BFB) (Fotovat et al. 2013, 2014, 2015). Singh et al. (2019) investigated the dynamics of segregation and fluidization of binary mixtures in a cylindrical fluidized bed using the measurements of solids volume fraction from the Electrical Capacitance Tomography (ECT). However, the above measurements need additional tracers and sophisticated instruments, which cannot be applied in actual industrial processes.

Electrostatic charges are generated on the solid particles during fluidization due to the contact and friction between particles, as well as the collisions between the particles and the wall. The charging characteristics of solid particles depend on many factors, including particle properties (size, work function and moisture content), environmental parameters (temperature and humidity) and flow hydrodynamics (gas velocity and solids concentration) (Fotovat et al. 2017). Although electrostatic phenomenon causes undesirable particle deposition and adhesion, measurement errors and even instrument malfunction (Gao et al. 2012; Li et al. 2015), it is widely used in measuring flow hydrodynamics of solid particles. Electrostatic sensors together with other types of sensors have been applied to measure mono-phase solid particles and particle mixtures in the pneumatic conveying pipeline because of the advantages of simple structure, low cost and high sensitivity (Qian and Yan 2012; Qian et al. 2015; Zhang et al. 2012; Wang et al. 2016). In recent years, the electrostatic sensor has also been applied to measure the flow behavior of solid particles in the fluidized bed. The hydrodynamics in the circulating fluidized bed (CFB) was investigated by the combined use of the electrostatic sensor and the ECT (Zhang et al. 2013). The flow behaviors and charging characteristics of Geldart A and D particles in the dense and transit regions of the bubbling bed were measured using the electrostatic sensor arrays (Zhang et al. 2016; Yang et al. 2017; Shi et al. 2017). However, there is no report on the application of the electrostatic sensor in the measurement of binary particle mixtures in the fluidized bed.

In this paper, the particle velocity of binary mixtures in the riser of a CFB is studied with the support of a ringshape electrostatic sensor. The cross-correlation calculation is applied to the electrostatic signals from upstream and downstream electrodes, during which a new type of correlation function is found. In order to further investigate the particle velocity measurement, a high-speed imaging system is designed to capture the flow images of the binary particle mixtures in the riser of the CFB and the velocity of each particle type in the mixtures is calculated by post-processing the images. Finally, the reasons for the new type correlation function are illustrated, and measured velocities from electrostatic sensing and high-speed imaging are compared. 


\section{Experimental setup}

Figure 1 shows the cold model of a lab-scale CFB on which the experimental work was undertaken. The lab-scale CFB consists of a riser with an inner diameter of $40 \mathrm{~mm}$ and a length of $4 \mathrm{~m}$, a downer with an inner diameter of $40 \mathrm{~mm}$ and a length of $2 \mathrm{~m}$, a gas-solid separator and a BFB $(150 \mathrm{~mm} \times 300 \mathrm{~mm}$ by $700 \mathrm{~mm}$ high). The material of the bed is acrylic. The experiments were conducted in the lab where the temperature and relative humidity were $28{ }^{\circ} \mathrm{C}$ and $40 \%$, respectively. The binary particle mixtures composed of polyethylene (PE) particles and inert particles of sand. The average diameter of PE particles is $2.7 \mathrm{~mm}$ and the density is $941 \mathrm{~kg} / \mathrm{m}^{3}$, while the average diameter and the density of sand particles are around $0.2 \mathrm{~mm}$ and $2650 \mathrm{~kg} /$ $\mathrm{m}^{3}$, respectively. The binary particle mixtures with different volume ratios (volume of PE particles/volume of sand particles), which are 3:1,1:1 and 1:3, respectively, were used in the experiments. The binary particle mixtures were fed into the BFB and then fluidized in the BFB for sufficiently long

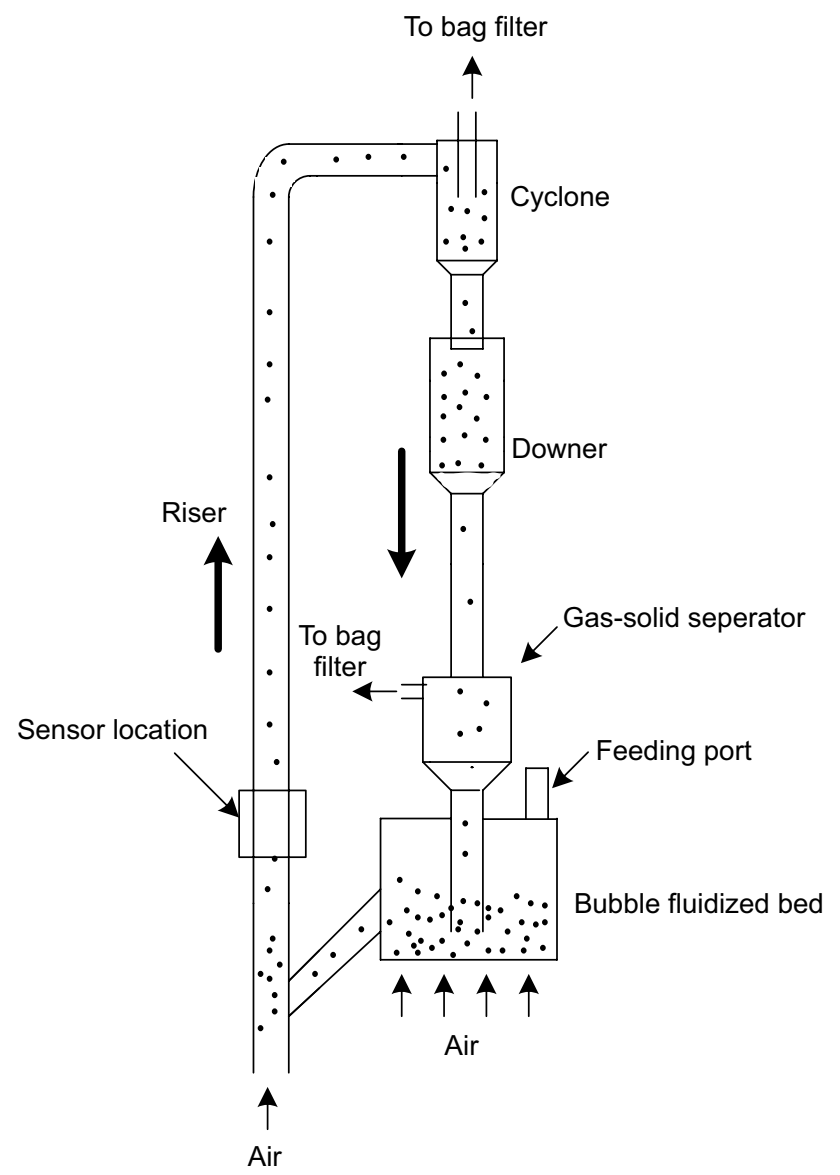

Fig. 1 Lab-scale circulating fluidized bed as a cold model experimental setup time (more than $20 \mathrm{~min}$ ) in order to achieve a homogenous mixing. Compressed air (about 5\% relative humidity) from an air compressor and a dryer was supplied at the bottom of the riser once the experiments started. The binary particles flowed down from the BFB to lower part of the riser because of gravity, and then were blown up along the riser owing to the compressed air flow. After passed through a smooth elbow, the particles entered a cyclone for gas-solid separation, where air escaped through the outlet of the cyclone and the solid mixtures moved down to the downer. Gas and solids were further separated by a separator at the outlet of downer, and finally the particles returned to the BFB. The range of air flow rate for present experimental setup is determined by the stable operation of the CFB and the superficial air velocity ranges from 9.3 to $13.3 \mathrm{~m} / \mathrm{s}$. With the increase of fluidization air velocity, there are different flow regimes in the riser, which are dense plug flow, core annular flow and dilute suspension flow, respectively. Since the flow was complex and turbulent at the riser inlet, the electrostatic sensor and the high-speed camera were placed at the height of $750 \mathrm{~mm}$ away from the riser inlet in order to minimize the influence of turbulence and focus on the dynamic behaviors of the fully developed flow. During the experiments, the electrostatic sensing and high-speed imaging were conducted at the same position in the riser under the same experimental conditions. After the electrostatic signals were obtained, the electrostatic sensor was replaced by a clear acrylic tube in order to capture to flow images of the particle mixtures under the same experimental conditions.

\section{Sensor design and measurement principle}

\subsection{Electrostatic sensing}

Charges are induced on the electrostatic electrodes when charged particles pass the nearby region. Because of the virtual earth on the signal conditioning circuit, there is an induced current on the electrodes (Zhang et al. 2013). The current signal from the sensor is transformed, filtered and amplified by using a signal conditioning circuit, as details given in reference (Zhang et al. 2016). The resulted electrostatic signal contains the information of the particle behaviors in the bed, by which further analysis can be conducted. The electrostatic sensor includes two ring-shape electrodes in the upstream and downstream of the flow, respectively, as shown in Fig. 2. Considering the similarity between the electrostatic signals and the variations of correlation results, the spacing between the upstream and downstream electrodes is set as $50 \mathrm{~mm}$. In consideration of the amplitude and bandwidth of the signal, the thickness and axial width of each electrode are $2 \mathrm{~mm}$ and $6 \mathrm{~mm}$, respectively. The electrodes are made of copper and are tightly wrapped 
(a)

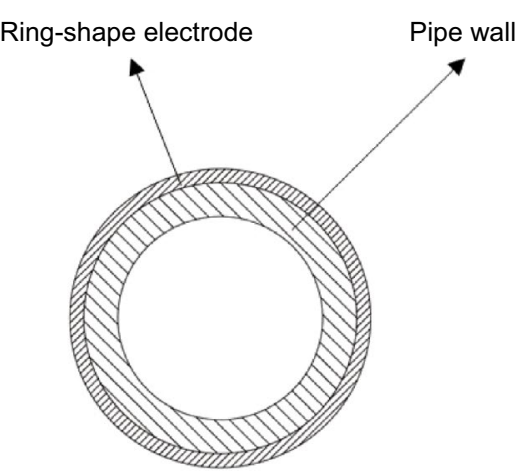

(b)

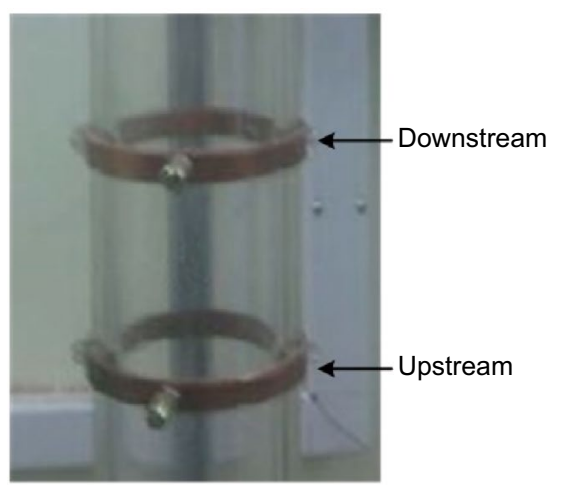

Fig. 2 Electrostatic sensor with ring-shape electrodes, a ring-shape electrode, b photo

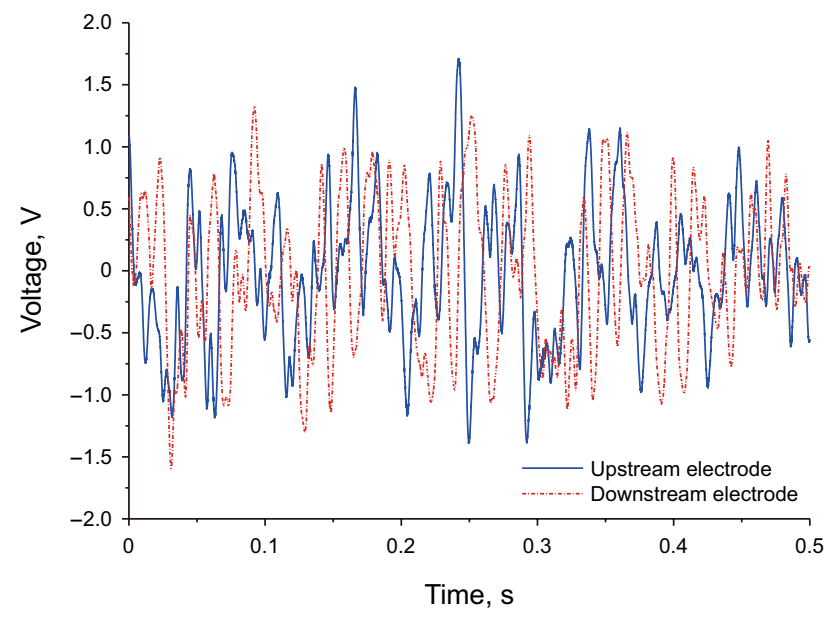

Fig. 3 Typical signals from the electrostatic sensor when particle mixtures with equal volume ratio flow together in the riser under the superficial air velocity of $13.3 \mathrm{~m} / \mathrm{s}$

around the outside surface of an acrylic pipe. The signal conditioning circuits together with the electrodes are shielded in a metal box to eliminate external noise. The amplified signals from the circuit boards are sampled simultaneously by using a data acquisition card and the resolution of the Analog to Digital Converter (ADC) is 12 bits. Sampling frequency of the electrostatic signal is $20 \mathrm{kHz}$ and the sampling duration is $60 \mathrm{~s}$. Typical signals from the electrostatic sensor when particle mixtures flow together in the riser are given in Fig. 3. It can be found that the electrostatic signal obtained in the downstream presents a similar pattern as the upstream signal, however, with around $0.01 \mathrm{~s}$ delay in time. The cross-correlation coefficient between these two signals is defined as:

$C(d)=\frac{1}{T} \int_{0}^{T} S_{1}\left(z_{1}, t\right) S_{2}\left(z_{2}, t+d\right) \mathrm{d} t$ where $C(d)$ is the cross-correlation coefficient and $T$ is the integral time. $S_{1}\left(z_{1}, t\right)$ and $S_{2}\left(z_{2}, t\right)$ are the upstream and downstream electrostatic signals, respectively. The correlation function is adopted to evaluate the similarity of the two signals and the time delay $(\tau)$ between the two signals is determined by the peak in the correlation function. The integral time $T$ in Eq. (1) is set to $0.5 \mathrm{~s}$ in this paper in order to reduce the uncertainty relating to the peak of the correlation function (Zhang et al. 2013). With the knowledge of the time delay and the distance between two electrodes, correlation velocity can be obtained. In the CFB system, although some particles move downwards in the wall region, the solid particles in the riser mainly move upwards in the remaining larger part of the cross section. Given the fact that the magnitude of upward velocity is remarkably greater than that of the downward velocity and the measured correlation velocity is always positive in the riser of the CFB, the correlation velocity is considered as the upward velocity of the particles. In addition, the charge on the particles is assumed at $\mathrm{nC}$ level based on the electrostatic signals from the sensor (Shi et al. 2017). As a result, the electrostatic effects do not influence the motion of particles in the riser.

\subsection{High-speed imaging}

In order to verify the results from the electrostatic sensing and analyze the particle velocities of binary mixtures, a high-speed imaging system was built to capture the flow images of binary mixtures in the CFB. The experimental setup is shown in Fig. 4, including a high-speed camera (Photron FASTCAM MC 2.1) and the lens (AF Micro Nikkor, focal length $60 \mathrm{~mm}$, maximum aperture $\mathrm{f} / 2.8$ ). Since the PE and sand particles are white in color, a black paper was attached at the back of the clear acrylic tube in order to make a good contrast. A high power halogen lamp (Unomat LX401GS) was used to illuminate the flow field inside the riser. The angle between the highspeed camera and the lamp was 45 degree. During the 


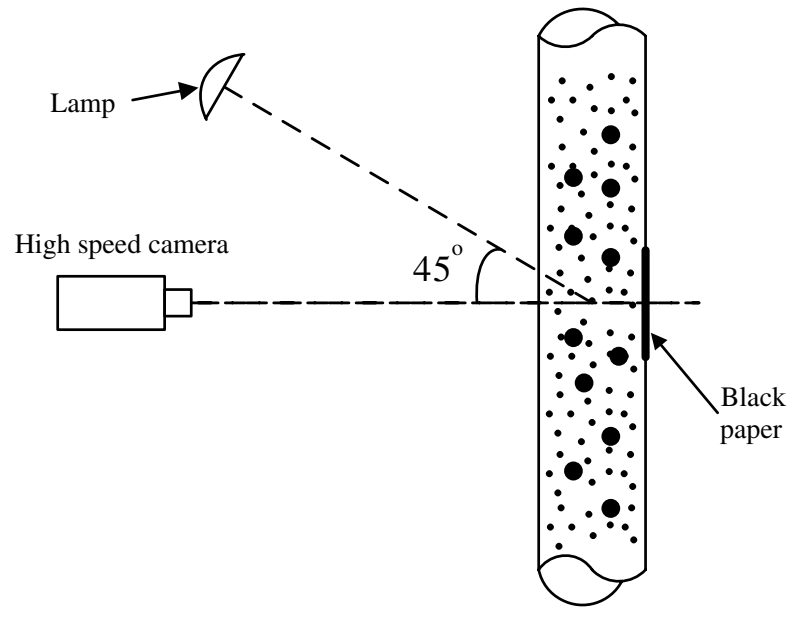

Fig. 4 Experimental setup of high speed imaging system

experiment, the frame rate of the high-speed camera was set as 3000 fps regarding to the high velocity of the solid particles and the video recording time was $4 \mathrm{~s}$. Totally 12,000 images were captured. The corresponding image resolution was $512 \times 352$ pixels. The shutter speed of the camera was set as $1 / 8000 \mathrm{~s}$ in order to avoid image blurring. Since the electrostatic sensor is more sensitive to the charged particles near the electrodes, the correlation velocities calculated from the electrostatic signals represent particle velocities near the pipe wall. The high-speed camera was adjusted to focus on the solid particles flowing near the pipe wall. Therefore, the particle velocities from the electrostatic sensing and high-speed imaging have the same physical meaning and they can be directly compared with each other. Depth of field of the camera was set as small as possible to eliminate the bad influence of the defocus particles in the images. Typical image obtained during the experiment is shown in Fig. 5, where $\mathrm{PE}$ and sand particles can be identified.

As shown in Fig. 5, due to the large difference in the particle diameter, PE particles present lower image density as compared to sand particles. According to the difference in the image density, the particle velocity of $\mathrm{PE}$ and sand particles will be analyzed in different ways. PE particles will be investigated using Particle Tracking Velocimetry (PTV) algorithm and sand particles will be analyzed using Particle Image Velocimetry (PIV) algorithm. The fundamental algorithms of PIV and PTV are different: PIV uses image correlation to calculate the velocity vectors of the particles, while PTV focuses on the tracking of the particle trajectory to calculate the velocities. The details of each algorithm are presented as following.

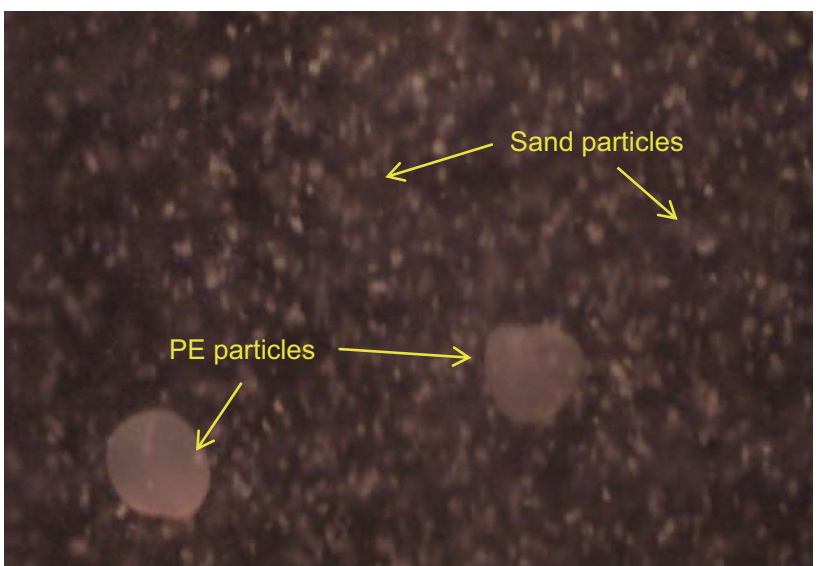

Fig. 5 Typical image (frame rate $3000 \mathrm{fps}$, resolution $512 \times 352$ pixels, shutter speed $1 / 8000 \mathrm{~s}$, superficial air velocity $11.97 \mathrm{~m} / \mathrm{s}$, particle mixture with equal volume ratio)

\subsubsection{PIV analysis}

PIV analysis in this paper was conducted by using a MATLAB toolbox named PIVlab 1.32 (Thielicke and Stamhuis 2014). The flow chart of the PIV algorithm is shown in Fig. 6. After the image series were loaded, image preprocessing was conducted to filter the images by enhancing the image contrast based on the contract-limited adaptive histogram equalization (Pizer et al. 1987). In addition, the function of drawing object mask is included in the toolbox. In this paper, the object mask was applied to PE particles in the image so as not to consider them in the PIV calculation of sand particles. The optimal particle density in PIV calculation is 5 to 15 particles per window and the interrogation area is adjusted according to the particle velocity by following a positive correlation (Thielicke and Stamhuis 2014). Particle velocity was obtained from sequential images based on direct cross-correlation (DCC), which has been proved to generate more accurate results than other approaches (Huang et al. 1997).

Due to the poorly illuminated regions in the image or strong out-of-plane flow, erroneous vectors might show up in the results. Therefore, vector validation was conducted to filter the erroneous data and the missing data were then interpolated. Velocity results were obtained by using the real distance and the time in the calibration. The acceptable velocities were selected to filter the outliners in the PIV results. The velocity thresholds were determined by comparing each velocity component with a lower threshold and an upper threshold ( $t_{\text {lower }}$ and $\left.t_{\text {upper }}\right)$ :

$t_{\text {lower }}=\bar{u}-n \times \sigma_{u}$

$t_{\text {upper }}=\bar{u}+n \times \sigma_{u}$ 


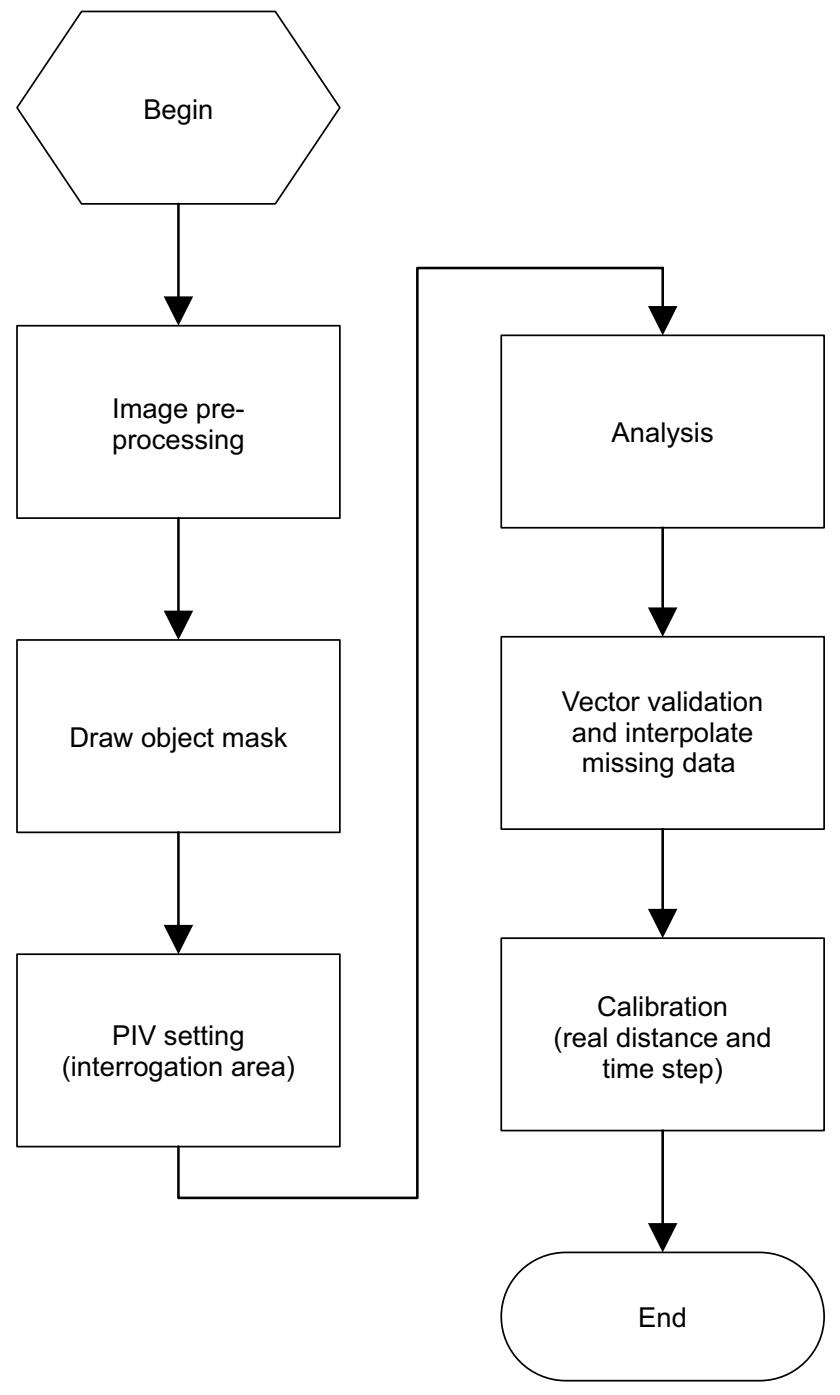

Fig. 6 Flow chart of PIV algorithm

where $\bar{u}$ is the mean velocity, $\sigma_{u}$ is the standard deviation of the velocities and $n$ is the strictness of this filter. The scatter plot of the velocity vectors is shown in Fig. 7 and in the figure $u$ and $v$ represent the radial and axial velocities of sand particles. The parameter $n$ in Eq. (2) is selected based on Fig. 7, which is set to 2 in this paper. Typical PIV result is shown in Fig. 8, where the two PE particles are masked and the vector map of sand particles is shown. It can be found from Fig. 8 that the sand particles mainly move upwards in the riser and there is no observable effect of PE particles on the measured sand velocities.

\subsubsection{PTV analysis}

In this paper, PTV analysis was conducted by using a selfdeveloped MATLAB code, which is based on the Image Processing Toolbox of MATLAB. The flow chart of the

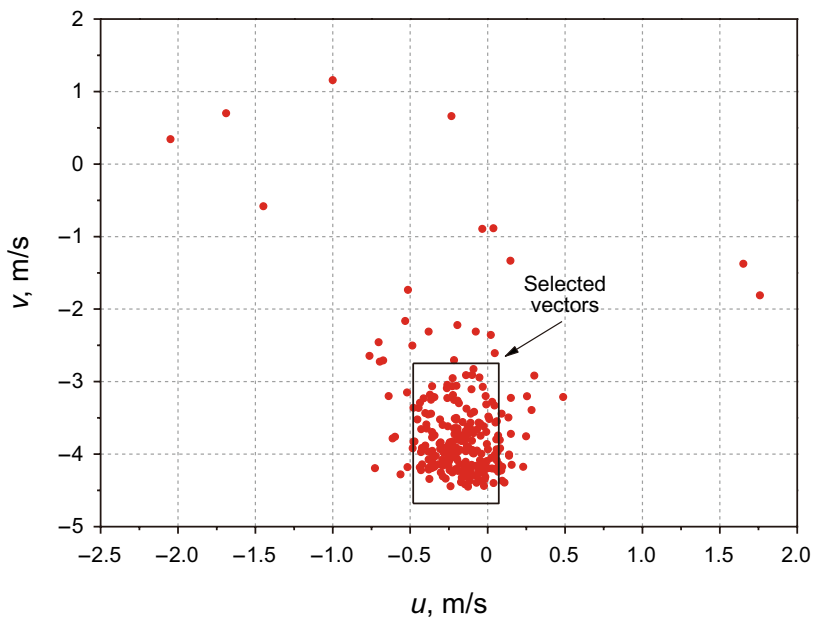

Fig. 7 Scatter plot of velocity vectors (interrogation area $48 \times 48$ pixels, superficial air velocity $11.97 \mathrm{~m} / \mathrm{s}$, particle mixture with equal volume ratio)

PTV algorithm is shown in Fig. 9. Three-step pre-processing was conducted to the image sequences of particle mixtures. Firstly, the image intensity values were adjusted and $1 \%$ of data is saturated at lowest and highest intensities of the image. This was to increase the image contrast and make the PE particles brighter in the output image. Secondly, the high frequency noise was eliminated by a median filter, a nonlinear operation that was effective to reduce "salt and pepper" noise. Thirdly, images were binarized. The pixel intensity which was greater than the threshold of 232 was set as the maximum value of 255 , with the rest being changed to zero. This threshold is chosen to remove the sand particles while remaining PE particles in the image. However, few sand particles are still left in the image owing to their high contrast. Therefore, following steps were carried out to

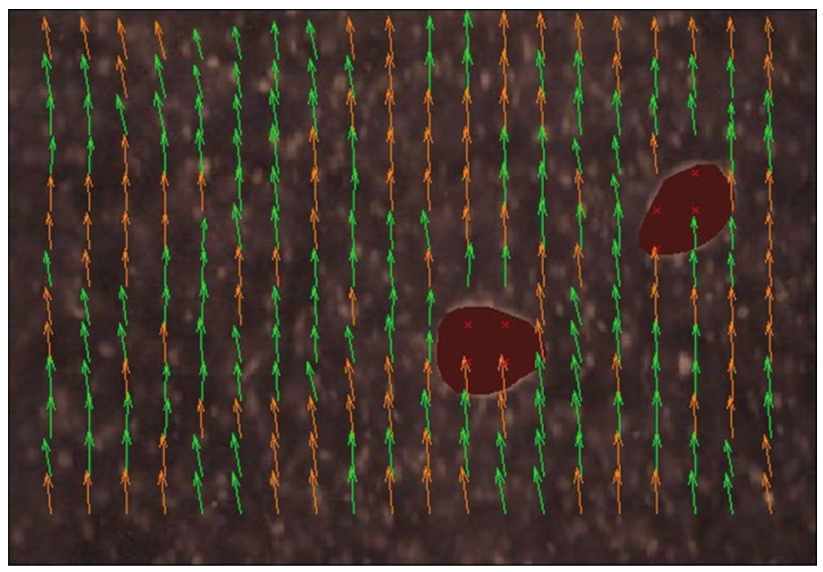

Fig. 8 Typical PIV result (interrogation area $48 \times 48$ pixels, superficial air velocity $11.97 \mathrm{~m} / \mathrm{s}$, particle mixture with equal volume ratio) 


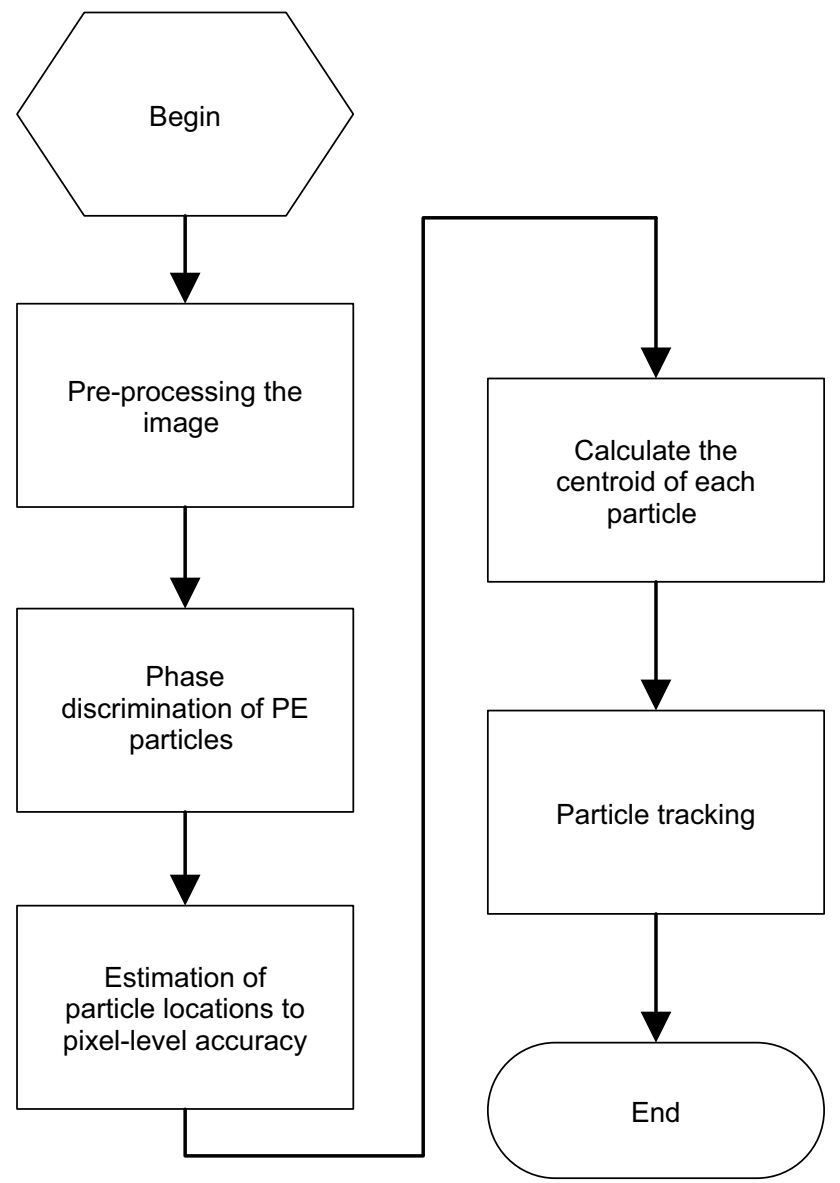

Fig. 9 Flow chart of PTV algorithm

keep PE particles only. Typical image after pre-processing is shown in Fig. 10a. Once the image was binarized, the contours of the connected domains were extracted to calculate their areas. The area threshold of 1200 was chosen for the phase discrimination of PE particles in the image. The connected domains with area greater than the threshold were treated as PE particles, whereas, the intensities in the rest regions were all set as zero for retaining PE particles only. The image after the phase discrimination is shown in Fig. 10b. The positions of PE particles can be identified after the image pre-processing and the phase discrimination, and the centroid of each particle is calculated by:

$m_{\mathrm{pq}}=\sum_{x=0}^{M-1} \sum_{y=0}^{N-1} x^{\mathrm{p}} y^{\mathrm{q}} f(x, y)$

$\bar{x}=\frac{m_{10}}{m_{00}} \bar{y}=\frac{m_{01}}{m_{00}}$

where $f(x, y)$ is the gray distribution of the binary image, $m_{\mathrm{pq}}$ is the 2-D moment of order $(\mathrm{p}+\mathrm{q})$, and $\bar{x}$ and $\bar{y}$ are the $x$ and $y$ coordinates of the centroid, respectively. The PE particles were tracked by identifying the pairs of particles with a minimal total squared displacement. The centroids of the PE particles from Fig. 10 in five subsequent images are represented in Fig. 11. It is shown that the lateral movement of the PE particles is not obvious compared to the movement in axial direction, indicating PE particles move upwards along with sand particles in the riser.

\section{Results and discussion}

\subsection{Electrostatic sensing result}

The electrostatic signals of the binary particle mixture fluidized in the riser were monitored and recorded under different superficial air velocities. Cross-correlation calculation was
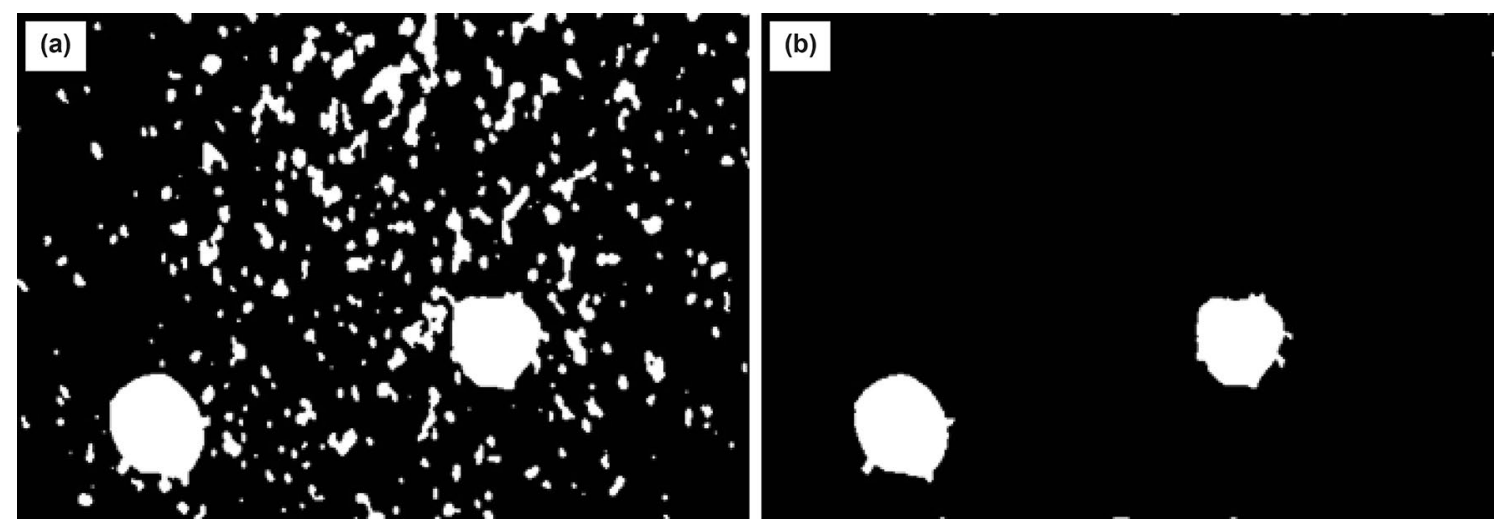

Fig. 10 Pre-processing and phase discrimination of the images, $\mathbf{a}$ image after pre-processing, $\mathbf{b}$ image after phase discrimination 
applied to the upstream and the downstream electrostatic signals. A new type of correlation function is found using Eq. (1) under different air velocities, as shown in Fig. 12. Two peaks present in the correlation function and the first peak and the second peak are close to each other. In addition, it is also found that correlation functions of electrostatic signals present two peaks when the particle mixtures with different volume ratios flowing together in the riser.

\subsection{Mono-solid phase study}

In order to further analyze the above results, experiments with mono-solid-phase were conducted when sand and PE particles were fluidized separately. By cross-correlating the electrostatic signals from upstream and downstream electrodes, only one-peak correlation function appeared, as shown in Fig. 13. Fast Fourier Transform (FFT) was applied to the electrostatic signals from the upstream ring-shape electrode. The frequency spectrums of the electrostatic signals of sand and PE particles are compared in Fig. 14. It is found that the electrostatic signal of sand particles has a higher bandwidth than that of PE particles under the same air velocity. The reason is that the flow behavior of sand particles in the riser is more turbulent because of the smaller size, resulting in more high frequency components in the electrostatic signal. In addition, the electrostatic signals from sand and PE particles have different frequency components, as shown in Fig. 14. As a consequence, it is supposed that the electrostatic signal contains two components when the PE and sand particles flow simultaneously: one is the signal from PE particles and the other is the signal from sand particles.

\subsection{Two-peak correlation function}

Based on the above analysis, the electrostatic signals from upstream and downstream electrodes are considered as:

$S_{1}\left(z_{1}, t\right)=S_{\text {sand }}\left(z_{1}, t\right)+S_{\mathrm{PE}}\left(z_{1}, t\right)$

$S_{2}\left(z_{2}, t\right)=S_{\text {sand }}\left(z_{2}, t\right)+S_{\mathrm{PE}}\left(z_{2}, t\right)$

where $S_{\text {sand }}(t)$ and $S_{\mathrm{PE}}(t)$ are the components from sand and PE particles, respectively, and $z_{1}$ and $z_{2}$ represent the upstream and downstream electrodes. The cross-correlation calculation of the upstream and downstream signals is reformulated as:

$$
\begin{aligned}
& C(d)=\frac{1}{T} \int_{0}^{T}\left(S_{\mathrm{sand}}\left(z_{1}, t\right)+S_{\mathrm{PE}}\left(z_{1}, t\right)\right)\left(S_{\mathrm{sand}}\left(z_{2}, t\right)+S_{\mathrm{PE}}\left(z_{2}, t\right)\right) \mathrm{dt} \\
& =\frac{1}{T} \int_{0}^{T}\left(S_{\mathrm{sand}}\left(z_{1}, t\right) S_{\mathrm{sand}}\left(z_{2}, t\right)+S_{\mathrm{sand}}\left(z_{1}, t\right) S_{\mathrm{PE}}\left(z_{2}, t\right)+S_{\mathrm{PE}}\left(z_{1}, t\right) S_{\mathrm{sand}}\left(z_{2}, t\right)+S_{\mathrm{PE}}\left(z_{1}, t\right) S_{\mathrm{PE}}\left(z_{2}, t\right)\right) \mathrm{dt} \\
& =\frac{1}{T} \int_{0}^{T} S_{\mathrm{sand}}\left(z_{1}, t\right) S_{\mathrm{sand}}\left(z_{2}, t\right) \mathrm{dt}+\frac{1}{T} \int_{0}^{T} S_{\mathrm{sand}}\left(z_{1}, t\right) S_{\mathrm{PE}}\left(z_{2}, t\right) \mathrm{dt}+\frac{1}{T} \int_{0}^{T} S_{\mathrm{PE}}\left(z_{1}, t\right) S_{\mathrm{sand}}\left(z_{2}, t\right) \mathrm{dt}+\frac{1}{T} \int_{0}^{T} S_{\mathrm{PE}}\left(z_{1}, t\right) S_{\mathrm{PE}}\left(z_{2}, t\right) \mathrm{dt}
\end{aligned}
$$

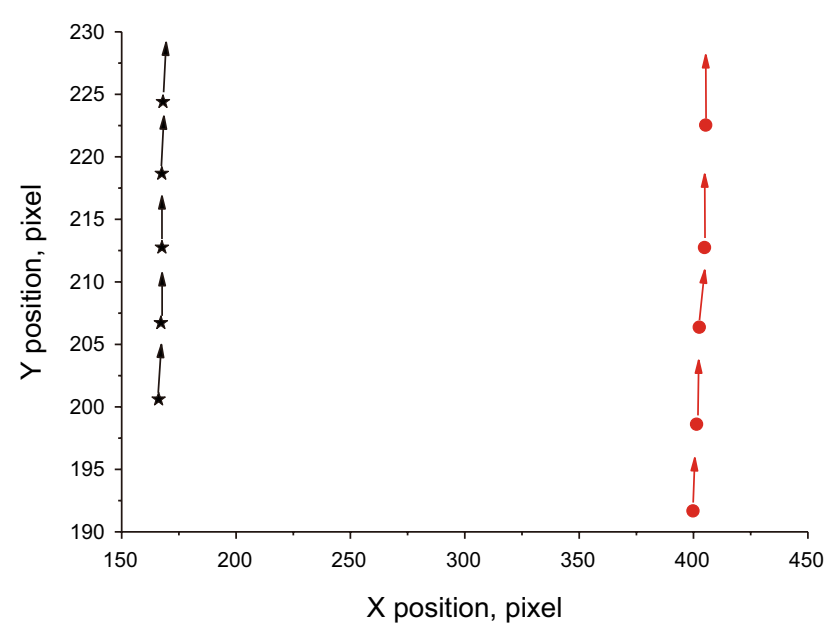

Fig. 11 Centroids of the PE particles from Fig. 10 in five consecutive images
It can be found that the correlation result is the sum of four correlation components. However, sand and PE components from the signals are not correlated mutually and the second and the third components in the correlation result will become zero when the integration time is long enough. As a result, the correlation result is the sum of the correlation components from the upstream and downstream sand and PE signals:

$C(d)=\frac{1}{T} \int_{0}^{T} S_{\text {sand }}\left(z_{1}, t\right) S_{\text {sand }}\left(z_{2}, t\right) \mathrm{dt}+\frac{1}{T} \int_{0}^{T} S_{\mathrm{PE}}\left(z_{1}, t\right) S_{\mathrm{PE}}\left(z_{2}, t\right) \mathrm{dt}$

As shown in Fig. 12, the two peaks in the correlation function are identified and the correlation velocity corresponding to each peak is calculated based on the time delay $\left(\tau_{1}\right.$ and $\tau_{2}$ ) and the distance $(L)$ between the upstream and the downstream electrodes, as given in Table 1. 

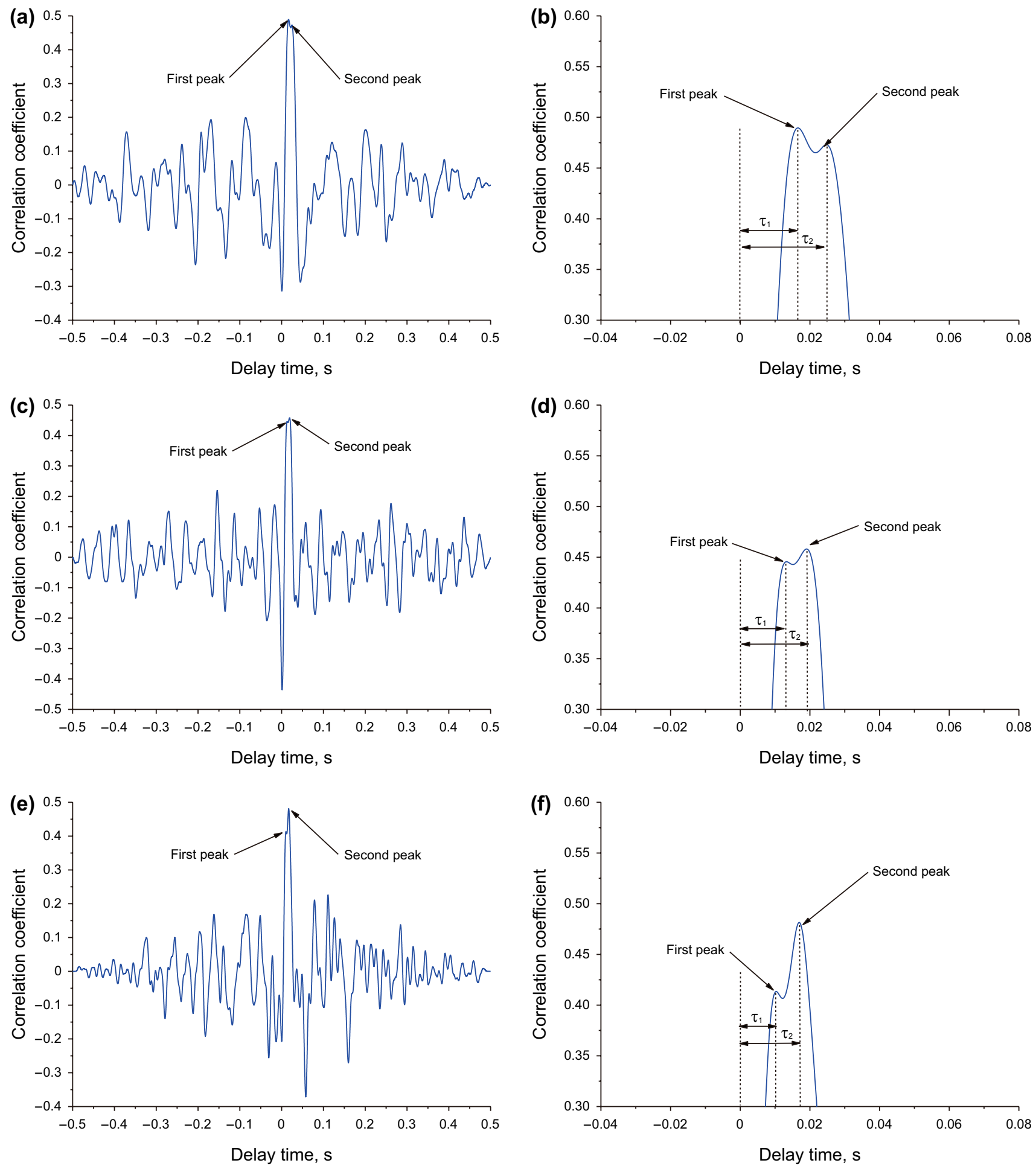

Fig. 12 Correlation function of electrostatic signals from particle mixture with equal volume ratio under different air velocities in the riser, a air velocity is $9.3 \mathrm{~m} / \mathrm{s}, \mathbf{b}$ zoom in version of $\mathbf{a}, \mathbf{c}$ air velocity is $10.64 \mathrm{~m} / \mathrm{s}, \mathbf{d}$ zoom in version of $\mathbf{c}, \mathbf{e}$ air velocity is $11.97 \mathrm{~m} / \mathrm{s}, \mathbf{f}$ zoom in version of $\mathbf{e}$

$v_{1}=\frac{L}{\tau_{1}}, v_{2}=\frac{L}{\tau_{2}}$

\subsection{High-speed imaging result}

The velocities of sand particles were calculated by following the PIV calculation algorithm given in Sect. 3.2.1. The 

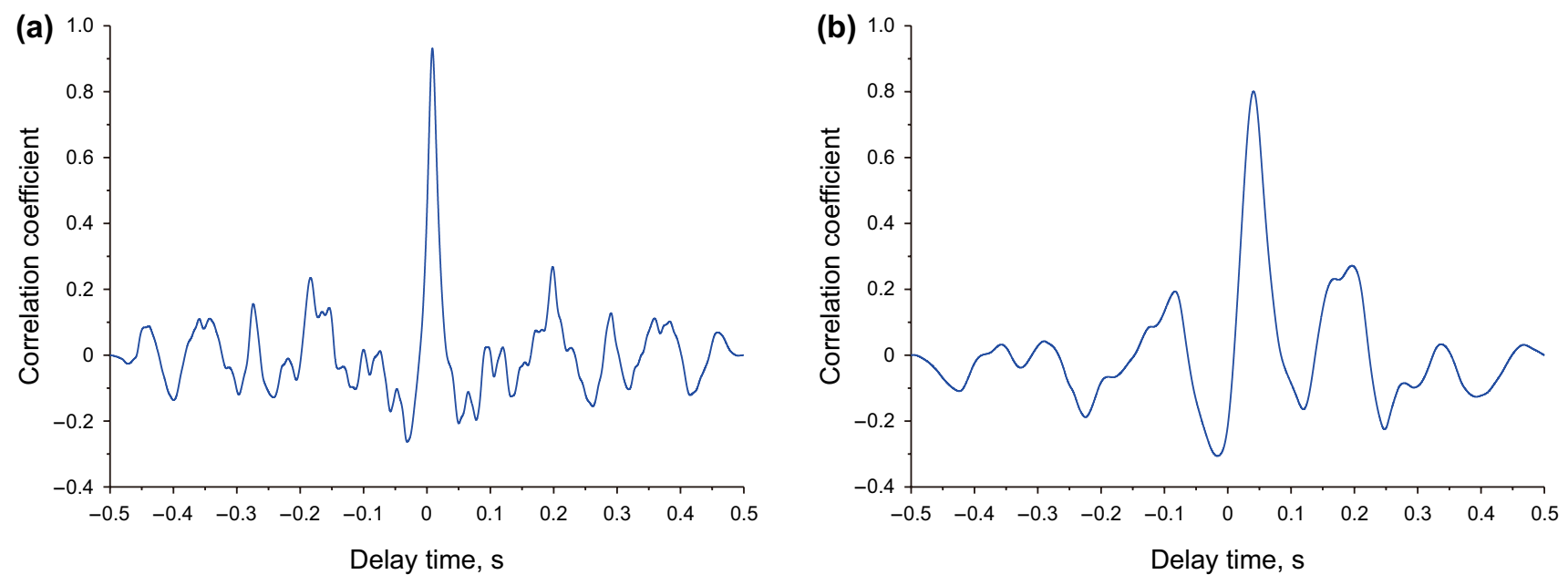

Fig. 13 Correlation function of the electrostatic signals when PE and sand are fluidized separately and the air velocity is $11.97 \mathrm{~m} / \mathrm{s}, \mathbf{a}$ sand, $\mathbf{b} \mathrm{PE}$

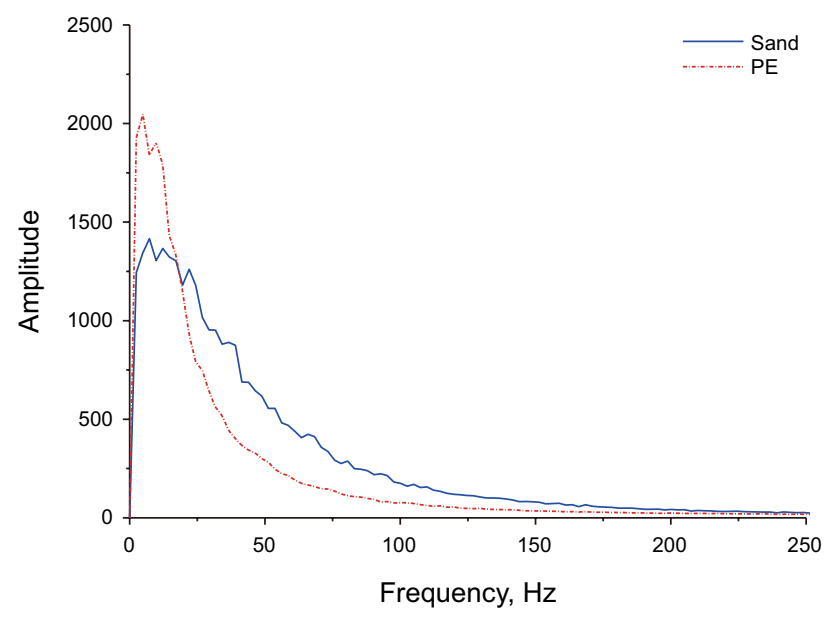

Fig. 14 Frequency spectrums of the electrostatic signals from upstream ring-shape electrode when PE and sand are fluidized separately and the air velocity is $11.97 \mathrm{~m} / \mathrm{s}$

accuracy of PIV calculation using PIVlab was extensively evaluated using synthetic images with known properties. It was proved that direct cross-correlation (DCC) between the corresponding images are able to produce accurate results. The mean axial velocity of sand particles is summarized in Table 2. The interrogation area was changed to $64 \times 64$ pixels as the air velocity was increased to $13.3 \mathrm{~m} / \mathrm{s}$, with the aim to include enough particles for analysis. It is demonstrated that the sand velocity increases with the air velocity in the riser and the stand deviations are low due to the fully developed flow.

Based on the frame rate of the images and the calibration result, which reflects the relationship between the pixel displacement and the real distance, the velocities of PE particles are calculated using the PTV calculation algorithm given in Sect. 3.2.2. The uncertainty of PTV calculation is reduced by averaging the results from 12,000 flow images. Table 3 summarizes the mean and standard deviation of PE axial velocity under different air velocities. It is found that the standard deviation of PTV results decreases with the increase of air velocity. The reason for the result is addressed to the different flow regimes in the riser. There exists obvious back mixing of PE particles in the riser under lower air velocity, which results in higher velocity fluctuation of $\mathrm{PE}$ particles. With the increase of air velocity, the flow regime in the riser changes to dilute suspension flow and the velocity fluctuation becomes lower.

Comparisons between Tables 2 and 3 show that the velocities of PE and sand particles are highly different. Particle Reynolds number $R e_{\mathrm{p}}$ for PE particle is larger than 1000 , the drag coefficient of which is in the Newton's regime. However, the $R e_{\mathrm{p}}$ for sand particle is nearly 100 , the drag coefficient of which is in the intermediate regime. Since drag force

Table 1 Correlation velocities from ring-shape electrodes

\begin{tabular}{|c|c|c|c|c|}
\hline Air velocity (m/s) & 13.3 & 11.97 & 10.64 & 9.3 \\
\hline Average of correlation velocity $1(\mathrm{~m} / \mathrm{s})$ & 5.221 & 5.003 & 4.162 & 3.46 \\
\hline Standard deviation of correlation velocity $1(\mathrm{~m} / \mathrm{s})$ & 0.389 & 0.28 & 0.367 & 0.361 \\
\hline Average of correlation velocity $2(\mathrm{~m} / \mathrm{s})$ & 2.751 & 2.569 & 2.414 & 1.978 \\
\hline Standard deviation of correlation velocity $2(\mathrm{~m} / \mathrm{s})$ & 0.107 & 0.49 & 0.239 & 0.127 \\
\hline
\end{tabular}


Table 2 Sand axial velocity of the particle mixture with equal volume ratio under different air velocities using PIV algorithm

\begin{tabular}{lllll}
\hline Air velocity, m/s & 13.3 & 11.97 & 10.64 & 9.3 \\
Interrogation area, pixels & $64 \times 64$ & $48 \times 48$ & $48 \times 48$ & $48 \times 48$ \\
$\begin{array}{l}\text { Mean sand axial velocity, m/s } \\
\text { Standard deviation of sand }\end{array}$ & 4.429 & 3.768 & 3.596 & 2.825 \\
$\quad$ axial velocity, m/s & & 0.087 & 0.013 & 0.02 \\
\hline
\end{tabular}

Table 3 PE axial velocity of particle mixture with equal volume ratio under different air velocities using PTV algorithm

\begin{tabular}{lcccl}
\hline Air velocity, m/s & 13.3 & 11.97 & 10.64 & 9.3 \\
Mean PE axial velocity, m/s & 3.113 & 2.766 & 2.296 & 1.834 \\
$\begin{array}{l}\text { Standard deviation of PE axial } \\
\text { velocity, m/s }\end{array}$ & 0.055 & 0.139 & 0.258 & 0.568 \\
\hline
\end{tabular}

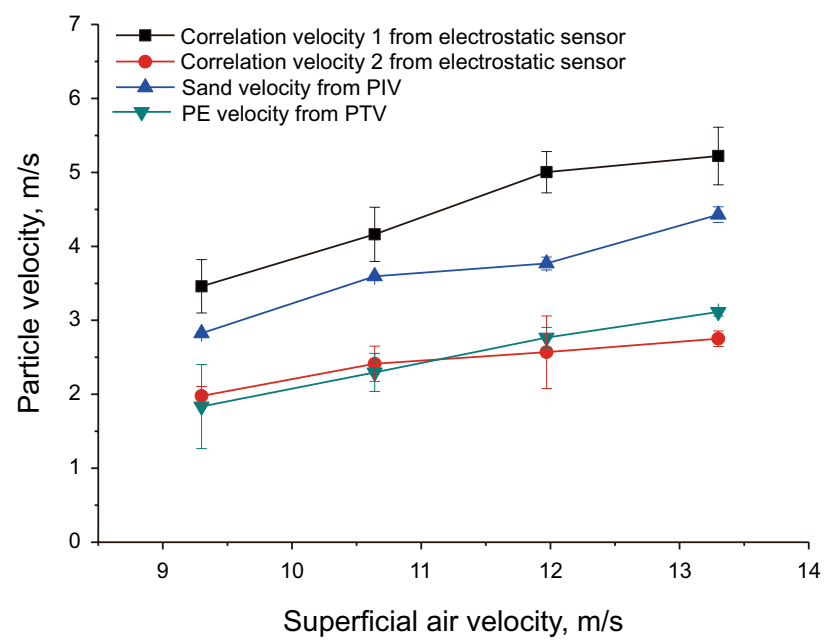

Fig. 15 Comparison of the results from electrostatic sensing and high-speed imaging (error bar in the image shows the standard deviation)

on the particle in the intermediate regime is much larger than that in the Newton's regime (Turton and Levenspiel 1986), the axial velocity of sand particles is larger than that of PE particles under the same air velocity. Finally, it is straightforward to deduce that the velocity difference between PE and sand particles in the riser results in the two-peak correlation function of the electrostatic signals.

\subsection{Comparison of results from electrostatic sensing and high-speed imaging}

The correlation velocities from the ring-shape electrostatic sensor are compared with the results from high-speed imaging, as shown in Fig. 15. It is known that the correlation velocity 2 from the sensor is comparable to the velocity of PE particles, with the maximum relative deviation less than $15 \%$. The correlation velocity 1 is higher than the result from PIV calculation. However, the maximum relative deviation between the two results is less than $25 \%$. The discrepancy of the two results may be caused by noise, particle pair loss and motion blur in the flow images, which results in the erroneous vectors in the PIV calculation. In addition, the flow behavior of sand particles in the riser is more turbulent and the high velocity fluctuation may results in the difference of velocity measurement. It is concluded that the two peaks in the correlation function of electrostatic signals correspond to the velocities of PE and sand particles, respectively. Although the two methods are based on different measurement principles, the electrostatic sensing and the high-speed imaging give comparable results of the velocities of PE and sand particles in binary particle mixtures.

\section{Conclusions}

The electrostatic sensor is applied to measure the flow behaviors of binary particle mixtures in the CFB. It is found that the correlation function of electrostatic signals presents two peaks when PE and sand particles flow together in the riser. In order to investigate the above result, high-speed imaging is applied to capture the flow images of the particle mixtures in the riser and the velocities of PE and sand particles are obtained by processing the flow images based on PTV and PIV algorithms. It is concluded that different components in the electrostatic signals and the significant difference between the velocities of PE and sand particles are the main reasons for the two-peak correlation function. In addition, the velocities of PE and sand particles are calculated from the two peaks in the correlation function of electrostatic signals. It is found that the velocity results from the electrostatic sensing and high-speed imaging are comparable. As a result, the electrostatic sensor can be applied to roughly estimate the particle velocity of binary particle mixtures in the near wall region of the CFB, which provides a low-cost online measurement method for the optimization of the related industrial processes.

Acknowledgements Zhang WB acknowledges the financial supports from the National Natural Science Foundation of China (No. 61403138) and Beijing Natural Science Foundation (No. 3202028). Zhan W and Wang CH acknowledge the research programme funded by the National Research Foundation (NRF), Prime Minister's Office, Singapore under its Campus for Research Excellence and Technological Enterprise (CREATE) programme. Grant Number R-706-001-102-281, National University of Singapore.

Open Access This article is licensed under a Creative Commons Attribution 4.0 International License, which permits use, sharing, adaptation, distribution and reproduction in any medium or format, as long 
as you give appropriate credit to the original author(s) and the source, provide a link to the Creative Commons licence, and indicate if changes were made. The images or other third party material in this article are included in the article's Creative Commons licence, unless indicated otherwise in a credit line to the material. If material is not included in the article's Creative Commons licence and your intended use is not permitted by statutory regulation or exceeds the permitted use, you will need to obtain permission directly from the copyright holder. To view a copy of this licence, visit http://creativecommons.org/licenses/by/4.0/.

\section{References}

Chen J, Lu X. Progress of petroleum coke combusting in circulating fluidized bed boilers - a review and future perspectives. Resour Conserv Recycl. 2007;49(3):203-16. https://doi.org/10.1016/j. resconrec.2006.03.012

Chew JW, Hays R, Findlay JG, Karri SBR, Knowlton TM, Cocco $\mathrm{RA}$ et al. Species segregation of binary mixtures and a continuous size distribution of Group B particles in riser flow. Chem Eng Sci. 2011a;66(20):4595-604. https://doi.org/10.1016/j. ces.2011.06.031

Chew JW, Parker DM, Cocco RA, Hrenya CM. Cluster characteristics of continuous size distributions and binary mixtures of Group B particles in dilute riser flow. Chem Eng J. 2011b;178:348-58. https://doi.org/10.1016/j.cej.2011.10.020

Cui J, Duan LB, Jiang Y, Zhao CS, Anthony EJ. Migration and emission of mercury from circulating fluidized bed boilers co-firing petroleum coke and coal. Fuel. 2018;215:638-46. https://doi. org/10.1016/j.fuel.2017.11.062

Duan LB, Cui J, Jiang Y, Zhao CS, Anthony EJ. Partitioning behavior of Arsenic in circulating fluidized bed boilers co-firing petroleum coke and coal. Fuel Process Technol. 2017;166:107-14. https:// doi.org/10.1016/j.fuproc.2017.05.003

Fotovat F, Chaouki J, Bergthorson J. The effect of biomass particles on the gas distribution and dilute phase characteristics of sandbiomass mixtures fluidized in the bubbling regime. Chem Eng Sci. 2013;102:129-38. https://doi.org/10.1016/j.ces.2013.07.042

Fotovat F, Chaouki J, Bergthorson J. Distribution of large biomass particles in a sand-biomass fluidized bed: experiments and modeling. AIChE J. 2014;60(3):869-80. https://doi.org/10.1002/aic.14337.

Fotovat F, Ansart R, Hemati M, Simonin O, Chaouki J. Sand-assisted fluidization of large cylindrical and spherical biomass particles: experiments and simulation. Chem Eng Sci. 2015;126:543-59. https://doi.org/10.1016/j.ces.2014.12.022

Fotovat F, Bi XT, Grace JR. Electrostatics in gas-solid fluidized beds: a review. Chem Eng Sci. 2017;173:303-34. https://doi. org/10.1016/j.ces.2017.08.001

Gao HM, Xu CL, Fu FF, Wang SM. Effects of particle charging on electrical capacitance tomography system. Measurement. 2012;45(3):375-83. https://doi.org/10.1016/j.measuremen t.2011.11.007

Gao JS, Chang J, Lu CX, Xu CM. Experimental and computational studies on flow behavior of gas-solid fluidized bed with disparately sized binary particles. Particuology. 2008;6(2):59-71. https ://doi.org/10.1016/j.partic.2007.11.003

Huang H, Dabiri D, Gharib M. On errors of digital particle image velocimetry. Meas Sci Technol. 1997;8(12):1427-40. https://doi. org/10.1088/0957-0233/8/12/007

Li J, Kong M, Xu CL, Wang SN, Wang SM. Influence of particle electrification on AC-based capacitance measurement and its elimination. Measurement. 2015;76:93-103. https://doi.org/10.1016/j. measurement.2015.08.017

Pizer SM, Amburn EP, Austin JD, Cromartie R, Geselowitz A, Greer $\mathrm{T}$ et al. Adaptive histogram equalization and its variations.
Comput Vis Graph Image Process. 1987;39:355-68. https://doi. org/10.1016/S0734-189X(87)80186-X

Qian XC, Yan Y. Flow measurement of biomass and blended biomass fuels in pneumatic conveying pipelines using electrostatic sensorarrays. IEEE T Instrum Meas. 2012;61(5):1343-52. https://doi. org/10.1109/TIM.2011.2175034

Qian XC, Yan Y, Wang LJ, Shao JQ. An integrated multi-channel electrostatic sensing and digital imaging system for the on-line measurement of biomass-coal particles in fuel injection pipelines. Fuel. 2015;151:2-10. https://doi.org/10.1016/j.fuel.2014.11.013

Ren AX, Wang TY, Tang TQ, He YR. Non-spherical particle mixing behaviors by spherical inert particles assisted in a fluidized bed. Petrol Sci. 2020;17:509-24. https://doi.org/10.1007/s12182-019-00401-4

Shi Q, Zhang Q, Han GD, Zhang WB, Wang JD, Huang ZL et al. Simultaneous measurement of electrostatic charge and its effect on particle motions by electrostatic sensors array in gas-solid fluidized beds. Powder Technol. 2017;312:29-37. https://doi. org/10.1016/j.powtec.2017.02.014

Singh BK, Roy S, Buwa VV. Dynamics of segregation and fluidization of binary mixtures in a cylindrical fluidized bed. AIChE J. 2019;65(10):e16682. https://doi.org/10.1002/aic.16682.

Sun JY, Yan Y. Non-intrusive measurement and hydrodynamics characterization of gas-solid fluidized beds: a review. Meas Sci Technol. 2016;27(11):112001. https://doi.org/10.1088/09570233/27/11/112001.

Thielicke W, Stamhuis E. PIVlab-towards user-friendly, affordable and accurate digital particle image velocimetry in matlab. J Open Res Softw. 2014;2(1):e30. https://doi.org/10.5334/jors.bl.

Turton R, Levenspiel O. A short note on the drag correlation for spheres. Powder Technol. 1986;47(1):83-6. https://doi. org/10.1016/0032-5910(86)80012-2.

Upadhyay RK, Roy S. Investigation of hydrodynamics of binary fluidized beds via radioactive particle tracking and dual-source densitometry. Can J Chem Eng. 2010;88(4):601-10. https://doi. org/10.1002/cjce.20334

Van Der Hoef MA, Ye M, van Sint AM, Andrews AT IV, Sundaresan S, Kuipers JAM. Multiscale modeling of gas-fluidized beds. Adv Chem Eng. 2006;31:65-149. https://doi.org/10.1016/S0065 $-2377(06) 31002-2$

Van Ommen JR, Mudde RF. Measuring the gas-solids distribution in fluidized beds-a review. Int J Chem React Eng. 2008;6:R3. https ://doi.org/10.2202/1542-6580.1796

Wang SN, Xu CL, Li J, Ding ZR, Wang SM. An instrumentation system for multi-parameter measurements of gas-solid twophase flow based on capacitance-electrostatic sensor. Measurement. 2016;94:812-27. https://doi.org/10.1016/j.measuremen t.2016.09.010

Yang Y, Zhang Q, Zi C, Huang ZL, Zhang WB, Liao ZW et al. Monitoring of particle motions in gas-solid fluidized beds by electrostatic sensors. Powder Technol. 2017;308:461-71. https://doi. org/10.1016/j.powtec.2016.11.034

Zhang J, Hu HL, Dong J, Yan Y. Concentration measurement of biomass/coal/air three-phase flow by integrating electrostatic and capacitive sensors. Flow Meas Instrum. 2012;24:43-9. https:// doi.org/10.1016/j.flowmeasinst.2012.03.003

Zhang WB, Cheng YP, Wang C, Yang WQ, Wang CH. Investigation on hydrodynamics of triple-bed combined circulating fluidized bed using electrostatic sensor and electrical capacitance tomography. Ind Eng Chem Res. 2013;52(32):11198-207. https://doi. org/10.1021/ie4009138

Zhang WB, Yan Y, Yang YR, Wang JD. Measurement of flow characteristics in a bubbling fluidized bed using electrostatic sensor arrays. IEEE T Instrum Meas. 2016;65(3):703-12. https://doi. org/10.1109/TIM.2016.2514698 